\title{
Cellular temperature compensation of sensory receptor neuron responses
}

\author{
Frederic A Roemschied ${ }^{1,2^{*}}$, Monika JB Eberhard ${ }^{3}$, Jan-Hendrik Schleimer ${ }^{1,2}$, Bernhard Ronacher ${ }^{2,3}$, \\ Susanne Schreiber ${ }^{1,2}$ \\ From Twenty Second Annual Computational Neuroscience Meeting: CNS*2013 \\ Paris, France. 13-18 July 2013
}

Temperature is known to modulate ion channel kinetics and hence also action-potential generation. This poses a challenge for neural systems that need to retain their functionality also under conditions of varying temperature. Multiple strategies to counterbalance the effects of environmental temperature changes exist: mammals keep their body temperature approximately constant, while poikilothermic species need to implement temperature-compensation at the behavioral, systems, or cellular level. While mechanisms of behavioral and systems level have been identified [1], cellular mechanisms of temperaturecompensation as well as their associated metabolic cost remain largely unknown.

We investigated the effect of temperature on auditory processing in the grasshopper. We recorded intracellular responses of auditory receptor neurons to auditory broad-band noise stimuli at different intensities at two distinct behaviorally relevant temperatures. Interestingly, we found that changes in temperature did not have large effects on sound-intensity coding in receptor neurons. These neurons constitute the input layer of a feedforward network and hence do not receive network input. We concluded that the observed temperature robustness of receptor-neuron responses must arise from intrinsic, network unrelated effects.

In general, the receptor-neuron response is shaped by two processing steps: mechanosensory transduction and spike generation. Both can contribute to temperature compensation. Either both transduction and spike generation are compensated (hypothesis I), or alternatively, their temperature dependencies can cancel each other (hypothesis II).

\footnotetext{
* Correspondence: frederic.roemschied@bccn-berlin.de

${ }^{1}$ Institute for Theoretical Biology, Humboldt-Universität zu Berlin, 10115 Berlin, Germany

Full list of author information is available at the end of the article
}

To test hypothesis I we assumed a temperature-invariant transduction and asked, first, whether temperature-compensation could be achieved for a spike-generating mechanism with realistic temperature dependencies of the ionic conductances. The latter refers, in particular, to increases of gating kinetics by a factor of 2-4 with temperature increments of $10^{\circ} \mathrm{C}$ (defining a Q10 value of 2-4) as well as modest increases of peak conductances. Second, we explored whether temperature compensation, if achieved cell-intrinsically, compromises the neuronal energy budget. In other words, is temperature robustness metabolically expensive? To address these questions, we varied the temperature dependence of ionic conductances in a conductance-based neuron model. Based on the spike frequency vs. input current (f-I) relation, we estimated the ability of the model neurons to keep a robust firing rate despite changing temperature. Moreover, we computed the average energetic cost per action potential [2]. Using a database modeling approach [3], we performed a systematic sensitivity analysis for firing-rate changes and energetic cost as a function of the temperature dependence of conductance parameters (i.e. Q10 values of transition rates and peak conductances). Our analysis shows that the key parameters determining the robustness of spike generation relate to the temperature-dependence of the model's potassium conductances. In contrast, energy consumption is governed by the temperature dependence of the sodium conductance. Consequently, a neuron can achieve temperature-compensation of its firing rate without compromising the energy budget.

To constrain hypothesis II, we used the experimentally observed f-I curves in an objective function and inferred the corresponding transduction process for each spike generation in our sensitivity analysis. Our results predict that thermosensitive Transient Receptor Potential (TRP) channels have a role in mechanosensory transduction at 
the grasshopper tympanum, and therefore motivate further experiments.

\section{Acknowledgements}

This work was supported by DFG (SFB 618, GRK 1589/1) and BMBF (BCCN Berlin, BPCN).

\section{Author details}

${ }^{1}$ Institute for Theoretical Biology, Humboldt-Universität zu Berlin, 10115 Berlin, Germany. ${ }^{2}$ Bernstein Center for Computational Neuroscience Berlin, Humboldt-Universität zu Berlin, 10115 Berlin, Germany. ${ }^{3}$ Department of Biology, Humboldt-Universität zu Berlin, 10115 Berlin, Germany.

Published: 8 July 2013

\section{References}

1. Robertson RM, Money TG: Temperature and neuronal circuit function: compensation, tuning and tolerance. Curr Opin Neurobiol 2012, 22:724-734.

2. Hasenstaub A, Otte S, Callaway E, Sejnowski TJ: Metabolic cost as a unifying principle governing neuronal biophysics. PNAS 2010, 107:12329-12334.

3. Prinz AA, Billimoria CP, Marder E: Alternative to Hand-Tuning Conductance-Based Models: Construction and Analysis of Databases of Model Neurons. J Neurophysiol 2003, , 90: 3998-4015.

\section{Submit your next manuscript to BioMed Central} and take full advantage of:

- Convenient online submission

- Thorough peer review

- No space constraints or color figure charges

- Immediate publication on acceptance

- Inclusion in PubMed, CAS, Scopus and Google Scholar

- Research which is freely available for redistribution

Submit your manuscript at www.biomedcentral.com/submit
C Biomed Central 